\title{
Flexiteste: proposição de cinco índices de variabilidade da mobilidade articular
}

\author{
Claudio Gil Soares de Araújo ${ }^{1}$
}

\section{RESUMO}

A flexibilidade pode ser definida como a máxima amplitude fisiológica passiva em um dado movimento articular. A flexibilidade é específica para a articulação e para o movimento. O Flexiteste, originalmente descrito em 1980, permite a medida da flexibilidade de 20 movimentos articulares, em uma escala crescente de números inteiros entre 0 e 4 , e a obtenção de um resultado global denominado de Flexíndice. Considerando que o mesmo Flexíndice pode ser obtido por diferentes combinações dos escores dos 20 movimentos, fazia-se necessário estudar de forma objetiva a variabilidade dos resultados. Utilizando resultados de 2.426 homens e mulheres entre 5 e 88 anos de idade, propomos cinco novos índices de variabilidade dos escores do Flexiteste: a) intermovimentos, b) interarticulações, c) flexão-extensão, d) entre segmentos, e e) distal-proximal. Os dois primeiros índices de variabilidade são independentes da idade, do gênero e da magnitude do Flexíndice. Os outros três, por ser resultados de quocientes entre médias de grupos de medidas, tendem a apresentar resultados próximos ao valor unitário, mas diferem entre os gêneros, especialmente quanto à dispersão dos resultados em relação à tendência central. Valores inferiores a 1,15 (homens) e 1,04 (mulheres) para o índice de variabilidade distal-proximal são pelo menos três vezes mais comuns em indivíduos com menos de 50 anos, sugerindo um padrão de expressão da flexibilidade infanto-juvenil. Métodos de cálculo, faixas de percentis para valores homogêneos, algo heterogêneos e altamente heterogêneos, assim como os aspectos princi-

1. Professor do Programa de Pós-Graduação em Educação Física da Universidade Gama Filho; Coordenador do Curso de Especialização em Medicina do Exercício e do Esporte da Universidade Estácio de Sá; Diretor Médico da Clínica de Medicina do Exercício (Clinimex) - Rio de Janeiro, RJ.

Recebido em: 25/9/2001

Aceito em: 14/1/2002

Endereço para correspondência:

E-mail: cgaraujo@iis.com.br pais da interpretação de cada um dos índices, são apresentados no texto.

Palavras-chave: Flexibilidade. Mobilidade articular. Lassitude ligamentar. Avaliação funcional. Cineantropometria.

\section{ABSTRACT}

\section{Flexitest: proposal of five variability indices for joint mo-} bility

Flexibility may be expressed as the maximum passive physiological range of motion in a given joint movement. Flexibility is specific to both joint and movement. The Flexitest, originally described in 1980, allows for the flexibility measurement of 20 joint movements, using a progressive scale of integer numbers from 0 to 4 , and provides a global score called Flexindex. Assuming that it is possible to obtain the same Flexindex by different combinations of scores in the 20 movements, an objective study of the variability of the results was required. Based on data obtained in 2,426 men and women ranging from 5 to 88 years of age, this paper proposes five new variability indices based on the scores from the Flexitest: $a$ ) intermovements, $b$ ) interjoints, c) flexion-extension, d) between segments, and e) distal-proximal. The two first indices do not depend on age, gender, and Flexindex magnitude. The other three, as a result of ratios between mean values of groups of measurements, tend to show values close to 1, while differing between genders, specially in relation to score dispersion around the central tendency. A distal-proximal variability index lower than 1.15 (men) and 1.04 (women) was observed at least three times more frequently in subjects younger than 50 years old, thus providing an infantile-juvenile pattern. Calculation methods, percentile ranges of scores for homogeneous, somewhat heterogeneous, and highly heterogeneous, as well as the major aspects of interpretation of each one of the indices are presented.

Key words: Flexibility. Joint mobility. Joint lassitude. Functional assessment. Kineanthropometry. 


\section{INTRODUÇÃO}

A flexibilidade é uma das principais variáveis da aptidão física relacionada à saúde ${ }^{1}$ e pode ser definida como a máxima amplitude fisiológica passiva em um dado movimento $\operatorname{articular}^{2}$. O grau de flexibilidade não é homogêneo em nosso corpo, variando, em realidade, para cada articulação e para cada movimento ${ }^{3}$. Ao final da década de 70 , trabalhando originalmente com o Prof. Roberto Pável, desenvolvemos um método adimensional de medida e avaliação da flexibilidade, que foi denominado de Flexiteste e apresentado originalmente em $1980^{4}$. A partir da rediagramação dos mapas de avaliação em $1986^{5}$ e em virtude de suas características, esse método tem sido, desde então, amplamente utilizado em situações de pesquisa ${ }^{5-7}$ e de prática profissional ${ }^{8,9}$ e veiculado em publicações nacionais ${ }^{10-12} \mathrm{e}$ estrangeiras ${ }^{9,13-15}$.

Em uma descrição sucinta, temos que o método consiste na medida e avaliação da mobilidade passiva máxima de 20 movimentos articulares corporais (36, se considerados bilateralmente), englobando as articulações do tornozelo, joelho, quadril, "tronco", punho, cotovelo e ombro. Oito movimentos são feitos nos membros inferiores, três no tronco e os nove restantes nos membros superiores. A numeração dos movimentos é feita em um sentido distal-proximal. Cada um dos movimentos é medido em uma escala crescente e descontínua de números inteiros de 0 a 4 , perfazendo um total de cinco valores possíveis. A medida é feita através da execução lenta do movimento até a obtenção do ponto máximo da amplitude e a posterior comparação entre as diversas posições dos mapas de avaliação e a amplitude máxima obtida pelo avaliador no avaliado. Habitualmente, o ponto máximo da amplitude de movimento é detectado com facilidade pela grande resistência mecânica à continuação do movimento e/ou pela informação de desconforto local pelo avaliado.

Pela natureza da escala e pelo modo como foram propositadamente desenhados os mapas de avaliação, observase distribuição praticamente gaussiana para os dados, de forma que a tendência central é o valor 2 , os valores 1 e 3 são menos freqüentes e os valores extremos, isto é, 0 e 4 , são bastante raros. Dessa forma, muito embora a análise do Flexiteste possa e deva ser feita para cada um dos movimentos e/ou articulações, é válido somar os resultados obtidos nos 20 movimentos isolados e obter um índice global de flexibilidade ou mobilidade articular, denominado de Flexíndice ${ }^{2,4,5,8,9}$, o que representa uma grande vantagem em relação à goniometria, na qual isso não é possível de ser feito. Em adendo, com a natureza gaussiana das escalas de cada movimento e do Flexíndice, é possível estudar todo o espectro da mobilidade, já que os valores extremos máximos - 0 e 80 pontos - nunca foram, na prática, obtidos. Dessa forma, não há os denominados efeitos solo e/ou teto, que tanto dificultam a utilização clínica de certos testes mais simples, especialmente os adimensionais ${ }^{16,17}$. Vários estudos comparando medidas intra e interobservadores em fotos de modelos ou em situações in vivo mostraram sistematicamente altos coeficientes de correlação intraclasse para o Flexiteste e, portanto, elevada fidedignidade ${ }^{2}$.

Muito embora o Flexíndice represente um escore global bastante interessante, é possível obter resultados idênticos partindo de diferentes combinações de escores para cada movimento. Na prática, não é incomum encontrarmos indivíduos com níveis extremos, para mais e para menos, de flexibilidade, isto é, a maioria dos movimentos com escores diferentes de 2 , e outros nos quais, praticamente, todos os movimentos apresentam mobilidade mediana e, portanto, escore 2, que, quando têm os seus Flexíndices calculados, apresentam resultados globais semelhantes. Isso parece ser relativamente comum quando comparamos atletas, por exemplo, voleibolistas, e indivíduos não-desportistas da mesma idade e gênero ${ }^{17}$. Parece, assim, oportuno, dentro da ótica de que a flexibilidade é especifica para articulação e para movimento, identificar índices capazes de representar a variabilidade dos escores dentro do Flexiteste e analisar seu comportamento em grande amostragem. Este artigo apresenta cinco novos índices que complementam a interpretação da variabilidade dos dados individuais do Flexiteste e que têm sido utilizados em nossa prática recente.

\section{METODOLOGIA}

\section{Amostra}

Os dados normativos para esses cinco índices foram obtidos em um banco de dados do Flexiteste composto de 2.426 indivíduos não-atletas, sendo 1.401 homens e 1.025 mulheres, entre 5 e 88 anos de idade. Todas as medidas foram colhidas por avaliadores experientes na metodologia do Flexiteste, sendo a maioria delas pelo próprio autor deste artigo.

\section{Índices de variabilidade para o Flexiteste}

Cinco novos índices de variabilidade adimensionais são propostos: a) intermovimentos, b) interarticular, c) flexão e extensão, d) entre segmentos, e e) distal-proximal. Para a interpretação dos índices, reconhecendo que as distribuições apresentadas são algo assimétricas, utilizamos as seguintes faixas de percentis: se menor do que $\mathrm{P}_{5}$ ou maior do que $\mathrm{P}_{95}$, temos um índice de variabilidade muito heterogêneo; se entre $\mathrm{P}_{5}$ e $\mathrm{P}_{17}$ ou entre $\mathrm{P}_{83}$ e $\mathrm{P}_{95}$, consideramos que o índice de variabilidade é algo heterogêneo; e se entre $\mathrm{P}_{17}$ e $\mathrm{P}_{83}$, correspondendo a um padrão homogêneo da flexibi- 
lidade corporal. Essas faixas equivalem a um $\left(\mathrm{P}_{17}\right.$ e $\left.\mathrm{P}_{83}\right)$ ou dois desvios padrões $\left(\mathrm{P}_{5}\right.$ e $\left.\mathrm{P}_{95}\right)$ em relação à média em uma amostra com distribuição gaussiana. Os dois primeiros índices não dependem da idade, do gênero ou da magnitude da flexibilidade. Os três últimos índices tendem a apresentar valores próximos à unidade e dependem da idade e do Flexíndice.

\section{Definições e fórmulas de cálculo}

\section{Índice de variabilidade intermovimentos (IVIM)}

A estatística mais freqüentemente utilizada para determinar a variabilidade de uma série de dados é a medida do desvio padrão. Essa estatística é capaz de calcular um escore diretamente relacionado às variações positivas ou negativas em torno da média enquanto mantém a escala original da medida. Analogamente, o IVIM é o valor do desvio padrão dos escores individuais dos 20 movimentos do Flexiteste.

\section{Índice de variabilidade interarticular (IVIA)}

O Flexiteste contempla 20 movimentos em sete articulações, aceitando a simplicidade de considerar o tronco como uma única articulação. Tal como foi feito para os 20 movimentos, é possível estudar a variabilidade das sete articulações. O IVIA representa o estudo da variabilidade entre as mobilidades médias obtidas nas diversas articulações. Para obter o IVIA, determinam-se inicialmente as médias dos escores para cada uma das articulações e a seguir calcula-se o desvio padrão entre essas médias, sem qualquer ponderação específica para o número de movimentos contemplados em cada articulação.

\section{Índice de variabilidade de flexão e extensão (IVFE)}

O IVFE reflete eventuais discrepâncias na mobilidade encontrada nos movimentos de flexão e de extensão articular. O IVFE é calculado a partir da divisão dos escores obtidos nos movimentos de flexão pela soma dos escores obtidos nos movimentos de extensão nas seis seguintes articulações: do tornozelo, joelho, quadril, tronco, punho e cotovelo.

\section{Índice de variabilidade entre segmentos (IVES)}

O IVES representa a comparação entre a mobilidade passiva dos segmentos inferior e superior do corpo. Para essa finalidade, obtém-se a média dos escores dos oito movimentos dos membros inferiores e divide-se pela média dos nove escores obtidos nos membros superiores.

\section{Índice de variabilidade distal-proximal (IVDP)}

O IVDP traduz eventuais discrepâncias entre a mobilidade passiva máxima das articulações distais e proximais dos

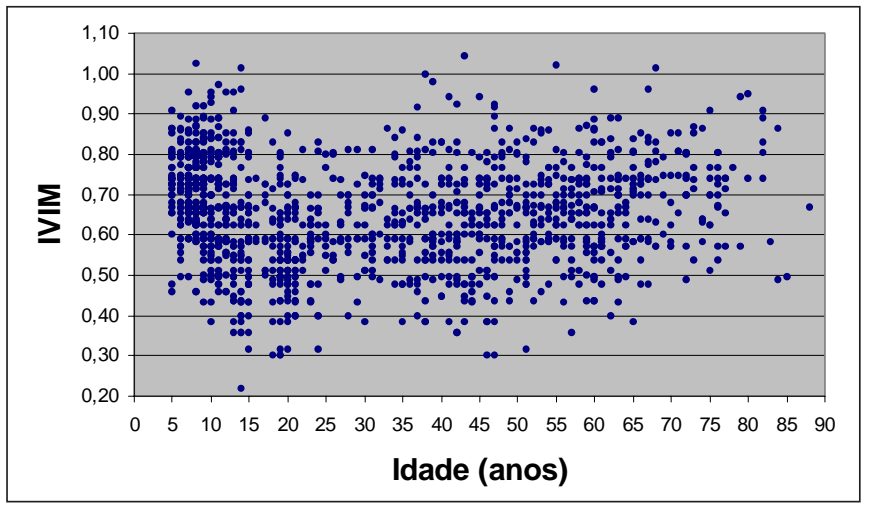

Fig. 1 - Relação entre o índice de variabilidade intermovimentos (IVIM) e a idade em 1.425 homens $\left(r^{2}=0,00015 ; p=1,00\right)$

membros. O IVDP é calculado pelo quociente entre a média dos oito escores dos movimentos distais - tornozelo, joelho, punho e cotovelo - e a média dos nove movimentos proximais - quadril e ombro - dos membros superiores e inferiores. Na raríssima situação em que os valores de todos os oito movimentos proximais correspondem a 0 , atribui-se um valor de 10 ao IVDP.

\section{RESULTADOS}

\section{Índice de variabilidade intermovimentos (IVIM)}

Embora teoricamente o IVIM possa assumir valores entre 0 e 2, ele raramente ultrapassa o valor de 1 . O valor médio de IVIM é de aproximadamente 0,65 , tanto para homens como para mulheres, encontrando-se $2 / 3$ dos indivíduos entre 0,54 e 0,78 . Um indivíduo que apresenta cinco escores 1, cinco escores 3 e dez escores 2 nos seus 20 movimentos e, portanto, Flexíndice de 40 pontos, possui um IVIM de 0,63. Interessantemente, não há qualquer associação entre o IVIM e a idade, com valores de coeficiente de determinação $\left(\mathrm{r}^{2}\right)$ iguais a $0(\mathrm{p}=1,00)$ (figura 1$)$. Também inexiste qualquer associação relevante entre IVIM e o Flexíndice, ou seja, valores de $\mathrm{r}^{2}$ de $0,004(\mathrm{p}=0,99)$. Sendo assim, o IVIM independe da idade, do gênero e do grau de flexibilidade.

\section{Índice de variabilidade interarticular (IVIA)}

Utilizando o banco de dados, observamos um IVIA de $0,41 \pm 0,13$ (média e desvio padrão), com valores mínimos de 0 e máximo de 0,84 e cerca de $70 \%$ dos resultados entre 0,25 e 0,55 . Novamente, os resultados médios de homens e mulheres são praticamente idênticos, diferindo apenas em 0,003 ponto, o que não possui qualquer significado prático. Interessantemente, não há qualquer associação entre o IVIA e a idade ou ainda com o Flexíndice, com valores de coeficiente de determinação $\left(\mathrm{r}^{2}\right)$ praticamente iguais a $0(\mathrm{p}$ $=1,00$ ). 


\section{Índice de variabilidade de flexão e extensão (IVFE)}

Embora, teoricamente, os resultados possam assumir valores positivos em uma escala muito ampla, na prática, a quase totalidade dos valores situa-se entre 0,5 e 2 . Há uma mínima e não significativa diferença entre homens e mulheres nesse índice. $O$ valor médio de IVFE é de 1,13, mas os desvios padrões diferem, sendo de 0,29 no sexo masculino e 0,21 no sexo feminino. Assim posto, temos que o valor do IVFE entre os percentis 17 e 83 dos homens ficará entre 0,93 e 1,30 e, nas mulheres, entre 1,00 e 1,31.

\section{Índice de variabilidade entre segmentos (IVES)}

Os valores médios de IVES são ligeiramente, porém significativamente, maiores nos homens - 1,07 $\pm 0,29$ [média \pm desvio padrão] - do que nas mulheres - 1,04 \pm 0,20. Todavia, a distribuição dos resultados difere ligeiramente da curva normal, de modo que a faixa de homogeneidade proposta é melhor definida pelos percentis 17 e 83 e situase entre 0,87 e 1,25 para os homens e 0,88 e 1,18 para as mulheres. Valores que não se situam na faixa entre $0,74 \mathrm{e}$ 1,64 para homens e 0,78 e 1,44 para mulheres são considerados como altamente heterogêneos. O menor valor obtido para o IVES foi de 0,38 e, o maior, de 4,13.

\section{Índice de variabilidade distal-proximal (IVDP)}

A tendência central e a variabilidade do IVDP diferem para os dois gêneros. Para homens, temos 1,15 $\pm 0,66$ [média \pm desvio padrão] e, para mulheres, obtivemos 1,04 \pm 0,40 . Os resultados variaram entre 0 e 10 , proporcionando uma distribuição assimétrica dos dados. Nesse caso, as faixas de padrão homogêneo, algo heterogêneo e muito heterogêneo foram obtidas a partir dos percentis $3,17,50,83 \mathrm{e}$ 97 para cada um dos gêneros, conforme mostra a tabela 1 (também são apresentados os valores para outros índices de variabilidade do Flexiteste).

Existe uma moderada correlação entre a magnitude do IVDP e a idade, tanto no sexo masculino como no feminino, com coeficientes de correlação significativos entre 0,26 e 0,36 . Interessantemente, são bastante raros (5 a $10 \%$ dos casos) valores superiores ao valor médio do IVDP entre crianças e adolescentes dos dois sexos, enquanto nos indivíduos com mais de 60 anos, mais de $60 \%$ deles possuem valores elevados de IVDP.

A tabela 1 apresenta as diversas faixas de percentis correspondentes aos cinco índices de variabilidade do Flexiteste propostos para indivíduos dos dois gêneros.

A tabela 2 apresenta diversos exemplos de escores para os 20 movimentos e para o Flexíndice e os resultados dos cinco índices de variabilidade propostos neste artigo.

\section{DISCUSSÃO}

\section{Índice de variabilidade intermovimentos (IVIM)}

O IVIM representa a variabilidade dos escores do Flexiteste nos diferentes movimentos. Se todos os escores são idênticos, não haverá variabilidade e o IVIM será igual a 0 . Já quando os resultados dos escores são extremados ao máximo, isto é, 10 medidas iguais a 0 e as outras 10 iguais

TABELA 1

Faixas de valores para interpretação dos índices de variabilidade do Flexiteste

\begin{tabular}{|c|c|c|c|c|c|c|}
\hline \multicolumn{7}{|c|}{ Masculino } \\
\hline Faixa & Percentis & IVIM & IVIA & IVFE & IVES & IVDP \\
\hline $\begin{array}{l}\text { Muito heterogêneo } \\
\text { Algo heterogêneo } \\
\text { Normal } \\
\text { Algo heterogêneo } \\
\text { Muito heterogêneo }\end{array}$ & $\begin{array}{c}<\mathrm{P}_{5} \\
\mathrm{P}_{5} \text { a } \mathrm{P}_{17} \\
\mathrm{P}_{17} \text { a } \mathrm{P}_{83} \\
\mathrm{P}_{83} \text { a } \mathrm{P}_{95} \\
\quad>\mathrm{P}_{95}\end{array}$ & $\begin{array}{c}<0,44 \\
0,44 \text { a } 0,54 \\
0,55 \text { a } 0,78 \\
0,79 \text { a } 0,85 \\
>0,85\end{array}$ & $\begin{array}{c}<0,21 \\
0,21 \text { a } 0,28 \\
0,29 \text { a } 0,54 \\
0,55 \text { a } 0,64 \\
>0,64\end{array}$ & $\begin{array}{c}<0,80 \\
0,80 \text { a } 0,93 \\
0,94 \text { a } 1,29 \\
1,30 \text { a } 1,71 \\
>1,71\end{array}$ & $\begin{array}{c}<0,74 \\
0,74 \text { a } 0,86 \\
0,87 \text { a } 1,25 \\
1,26 \text { a } 1,64 \\
>1,64\end{array}$ & $\begin{array}{c}<0,74 \\
0,74 \text { a } 0,87 \\
0,88 \text { a } 1,30 \\
1,31 \text { a 2,09 } \\
>2,09\end{array}$ \\
\hline \multicolumn{7}{|c|}{ Feminino } \\
\hline Faixa & Percentis & IVIM & IVIA & IVFE & IVES & IVDP \\
\hline $\begin{array}{l}\text { Muito heterogêneo } \\
\text { Algo heterogêneo } \\
\text { Normal } \\
\text { Algo heterogêneo } \\
\text { Muito heterogêneo }\end{array}$ & $\begin{array}{c}<\mathrm{P}_{5} \\
\mathrm{P}_{5} \text { a } \mathrm{P}_{17} \\
\mathrm{P}_{17} \text { a } \mathrm{P}_{83} \\
\mathrm{P}_{83} \text { a } \mathrm{P}_{95} \\
>\mathrm{P}_{95}\end{array}$ & $\begin{array}{c}<0,48 \\
0,48 \text { a } 0,54 \\
0,55 \text { a } 0,79 \\
0,80 \text { a } 0,88 \\
>0,88\end{array}$ & $\begin{array}{c}<0,21 \\
0,21 \text { a } 0,28 \\
0,29 \text { a } 0,54 \\
0,55 \text { a } 0,64 \\
>0,65\end{array}$ & $\begin{array}{c}<0,83 \\
0,83 \text { a } 1,00 \\
1,01 \text { a } 1,29 \\
1,30 \text { a } 1,51 \\
>1,51\end{array}$ & $\begin{array}{c}<0,78 \\
0,78 \text { a } 0,88 \\
0,89 \text { a } 1,18 \\
1,19 \text { a } 1,44 \\
>1,44\end{array}$ & $\begin{array}{c}<0,75 \\
0,75 \text { a } 0,83 \\
0,84 \text { a } 1,13 \\
1,14 \text { a } 1,74 \\
>1,74\end{array}$ \\
\hline
\end{tabular}


TABELA 2

Resultados do Flexiteste e dos índices de variabilidade*

\begin{tabular}{|c|c|c|c|c|c|c|c|c|c|c|c|c|c|c|c|c|c|c|c|c|}
\hline Item & Movimento & & & & & & & & & & & & & & & & & & & \\
\hline ॥ & Tornozelo/flexão plantar & 0 & 1 & 0 & 2 & 2 & 2 & 2 & 2 & 1 & 0 & 1 & 2 & 1 & 1 & 1 & 3 & 1 & 3 & 2 \\
\hline V & Quadril/flexão & 0 & 1 & 0 & 2 & 2 & 2 & 2 & 2 & 2 & 0 & 1 & 2 & 1 & 2 & 1 & 1 & 1 & 3 & 2 \\
\hline III & J oelho/flexão & 0 & 1 & 0 & 2 & 2 & 2 & 2 & 2 & 2 & 0 & 1 & 2 & 1 & 2 & 2 & 3 & 1 & 3 & 2 \\
\hline $\mathrm{XI}$ & Tronco/flexão lateral & 0 & 1 & 1 & 2 & 2 & 2 & 2 & 2 & 2 & 1 & 1 & 2 & 2 & 2 & 2 & 2 & 2 & 2 & 2 \\
\hline $\mathrm{XVII}$ & Ombro/extensão e adução posterior & 0 & 1 & 1 & 2 & 2 & 2 & 2 & 2 & 2 & 1 & 1 & 2 & 2 & 2 & 2 & 1 & 2 & 1 & 2 \\
\hline$X V I I I$ & Ombro/extensão posterior & 0 & 1 & 1 & 2 & 2 & 2 & 2 & 2 & 2 & 2 & 1 & 2 & 2 & 2 & 2 & 1 & 2 & 1 & 2 \\
\hline XIX & Ombro/rotação lateral & 0 & 1 & 1 & 2 & 2 & 2 & 2 & 2 & 2 & 2 & 1 & 2 & 2 & 2 & 2 & 1 & 2 & 1 & 2 \\
\hline$X X$ & Ombro/rotação medial & 4 & 3 & 3 & 2 & 2 & 2 & 2 & 2 & 2 & 2 & 2 & 3 & 2 & 2 & 2 & 1 & 2 & 1 & 2 \\
\hline VIII & Quadril/sbdução & 4 & 3 & 3 & 2 & 2 & 2 & 2 & 2 & 2 & 2 & 2 & 3 & 2 & 2 & 2 & 1 & 2 & 3 & 2 \\
\hline XIII & Punho/extensão & 4 & 3 & 4 & 2 & 2 & 2 & 2 & 2 & 2 & 4 & 2 & 3 & 3 & 2 & 2 & 3 & 3 & 1 & 2 \\
\hline XIV & Cotovelo/flexão & 4 & 3 & 4 & 2 & 2 & 2 & 2 & 2 & 2 & 4 & 2 & 3 & 3 & 2 & 2 & 3 & 1 & 1 & 2 \\
\hline$X V$ & Cotovelo/extensão & 4 & 3 & 4 & 2 & 2 & 2 & 2 & 2 & 3 & 4 & 2 & 3 & 3 & 3 & 3 & 3 & 3 & 1 & 2 \\
\hline \multirow[t]{6}{*}{ IV } & J oelho/extensão & 4 & 3 & 4 & 1 & 3 & 3 & 4 & 2 & 3 & 4 & 2 & 3 & 3 & 3 & 3 & 3 & 3 & 3 & 2 \\
\hline & IVIM & 2,00 & 1,00 & 1,58 & 0,30 & 0,32 & 0,30 & 0,63 & 0,44 & 0,45 & 1,41 & 0,50 & 0,50 & 0,77 & 0,45 & 0,50 & 0,92 & 0,77 & 0,92 & 0,00 \\
\hline & IVIA & 1,34 & 0,67 & 1,32 & 0,23 & 0,27 & 0,23 & 0,53 & 0,35 & 0,46 & 1,23 & 0,33 & 0,33 & 0,70 & 0,46 & 0,47 & 0,88 & 0,00 & 0,93 & 0,00 \\
\hline & IVFE & 1,00 & 1,00 & 0,85 & 1,20 & 1,00 & 0,86 & 1,00 & 1,20 & 0,85 & 0,71 & 1,00 & 1,00 & 0,92 & 0,85 & 0,77 & 1,00 & 0,33 & 1,00 & 1,00 \\
\hline & IVES & 0,56 & 0,75 & 0,45 & 0,88 & 1,00 & 1,13 & 1,00 & 0,88 & 0,89 & 0,45 & 0,83 & 0,89 & 0,59 & 0,89 & 0,83 & 1,06 & 1,00 & 3,00 & 1,00 \\
\hline & IVDP & 1,41 & 1,19 & 1,50 & 0,88 & 1,00 & 1,13 & 1,00 & 0,88 & 1,00 & 1,34 & 1,13 & 1,07 & 1,19 & 1,00 & 1,06 & 3,00 & 1,00 & 1,06 & 1,00 \\
\hline
\end{tabular}

Flexíndice

$\begin{array}{lllllllllllllllllll}40 & 40 & 40 & 38 & 40 & 42 & 40 & 38 & 40 & 40 & 30 & 50 & 40 & 40 & 39 & 39 & 40 & 39 & 40\end{array}$

* IVIM - índice de variabilidade intermovimentos, IVIA - índice de variabilidade interarticular, IVFE - índice de variabilidade de flexão e extensão, IVES - índice de variabilidade entre segmentos, IVDP - índice de variabilidade distal-proximal. Medidas variam entre 0 e 4 pontos.

a 4, o valor do IVIM será igual a 2; portanto, o índice pode variar entre 0 e 2 . Portanto, um valor de IVIM de 0 indica que todos os escores foram idênticos, enquanto valores mais altos representam variabilidades maiores.

O IVIM, como seria desejável, é mais afetado por escores individuais muito extremos, isto é, 0 e 4 . Em um indivíduo com 18 escores individuais iguais a 2 e com apenas dois movimentos com resultados extremos, o IVIM já se aproxima da média populacional de 0,63 , exatamente o mesmo valor que é obtido pela combinação de oito escores menos extremados, isto é, 1 e 3 , com 12 escores iguais a 2 . Na prática, considerando um indivíduo com Flexíndice de 40 pontos, cada valor extremo encontrado equivale a quatro escores menos extremados para efeito do cálculo do IVIM. Para um Flexíndice próximo a 40, um IVIM tipicamente médio é obtido quando ocorrem entre cinco e 10 movimentos diferentes, cada um deles diferindo em um ponto do escore 2, ou quando temos entre um e quatro escores extremos em relação ao médio.
Um IVIM aumentado sinaliza que alguns movimentos são bem mais flexíveis do que outros, o que pode ser fruto de algum trabalho físico específico prévio ou de alguma lesão ou seqüela locomotora importante. Muitas vezes, valores de IVIM elevados são associados com longa prática de determinada modalidade desportiva e podem estar relacionados a algum padrão biomecânico específico de desempenho.

\section{Índice de variabilidade interarticular (IVIA)}

O IVIA representa a variabilidade na mobilidade entre as diversas articulações testadas no Flexiteste. Se todos os escores são idênticos, não haverá variabilidade e o IVIA será igual a 0 . No caso de os resultados de todos os movimentos de quatro das articulações serem máximos, isto é, iguais a 4, e nas outras três articulações os escores forem 0 , teremos a maior variabilidade possível e um valor de IVIA de praticamente 2 . Sendo assim, o IVIA varia entre $0 \mathrm{e}$ 2. Tal como ocorreu com o IVIA, um IVIM igual a 0 signifi- 
ca que todos os escores foram idênticos, enquanto valores mais altos representam variabilidades maiores. O IVIA, como esperado, é também bastante afetado por valores díspares de flexibilidade nas diversas articulações.

\section{Índice de variabilidade de flexão e extensão (IVFE)}

Um valor de IVFE elevado reflete predomínio da mobilidade passiva dos movimentos de flexão sobre os de extensão, enquanto valores baixos representam o inverso. Quando há equivalência na mobilidade de flexão e extensão, tendemos ao valor unitário. Quando o IVFE está isoladamente aumentado, temos predomínio da mobilidade de flexão em detrimento da extensão, o que na maioria das vezes está associado a perda de tonicidade muscular e a rigidez articular importantes, como freqüentemente acontece em indivíduos idosos sarcopênicos. Já em presença de um valor de IVFE diminuído, temos predomínio da mobilidade de extensão sobre a de flexão, o que é proporcionalmente menos comum.

\section{Índice de variabilidade entre segmentos (IVES)}

$\mathrm{O}$ valor do IVES tende à unidade. Quando ele se afasta apreciavelmente desse valor, seja para mais ou para menos, tem-se uma diferença importante na flexibilidade dos dois segmentos. Escores de IVES elevados representam predomínio da mobilidade de tornozelo, joelho e quadril, enquanto valores baixos identificam predomínio da mobilidade do punho, cotovelo e ombro. Essas discrepâncias podem significar padrões de uso específico, como em determinadas modalidades desportivas, ou ser decorrência de quadros patológicos, como, por exemplo, paraplegia, em que tende a haver maior mobilidade das articulações em uso em detrimento daquelas dos membros inferiores que não são tão mobilizadas.

\section{Índice de variabilidade distal-proximal (IVDP)}

Um valor alto de IVDP indica que a mobilidade passiva nos movimentos articulares distais - tornozelo, joelho, punho e cotovelo - excede apreciavelmente aquela observada nos movimentos das articulações mais proximais dos

\section{REFERÊNCIAS}

1. Araújo DSMS, Araújo CGS. Aptidão física, saúde e qualidade de vida relacionada à saúde. Rev Bras Med Esporte 2000;6:194-203.

2. Araújo CGS. Medida e avaliação da flexibilidade: da teoria à prática. Universidade Federal do Rio de Janeiro, Instituto de Biofísica. Tese de doutoramento, 1987:440p.

3. Dickinson RV. The specificity of flexibility. Res Q 1968;39:792-4.

4. Pável RC, Araújo CGS. Flexiteste - Nova proposição para avaliação da flexibilidade. In: Anais do Congresso Regional de Ciências do Esporte, Volta Redonda, 1980. membros, isto é, quadril e ombro. Conforme mencionado anteriormente, valores inferiores à média no IVDP, isto é, 1,15 e 1,04 , respectivamente, em homens e mulheres, caracterizam um padrão infanto-juvenil de flexibilidade passiva, em que a mobilidade do ombro e do quadril é bastante alta. Com o passar dos anos e notadamente após a quinta década de vida, o IVDP tende a aumentar consideravelmente, especialmente em função da expressiva perda da mobilidade passiva dos ombros que ocorre nos indivíduos mais idosos dos dois sexos. Por exemplo, 54\% dos homens e $75 \%$ das mulheres com mais de 50 anos de idade apresentaram valores de IVDP maiores do que 1,15 (homens) e 1,04 (mulheres), enquanto apenas cerca de 19\% dos homens mais jovens e $25 \%$ das mulheres mais jovens possuíam esse padrão de variabilidade.

\section{CONCLUSÃO}

Ao melhor do nosso conhecimento, a proposta de índices de variabilidade para estudo do perfil individual da flexibilidade é original. O grau de homogeneidade dos escores das medidas de mobilidade articular, avaliado pelos índices específicos - IVIM, IVIA, IVFE, IVES e IVDP -, reveste-se da maior importância para a interpretação dos resultados individuais do Flexiteste. Quanto maior a heterogeneidade das medidas, mais provável é a existência de limitações importantes de mobilidade articular a serem especificamente estudadas e trabalhadas ou perfis de excepcionalidade a serem explorados em gestos ou modalidades desportivas ou de dança. Os cinco índices de variabilidade propostos neste artigo complementam a análise da flexibilidade estática passiva máxima feita pelo Flexiteste, ampliando o uso clínico e desportivo das medidas da mobilidade articular no âmbito da Medicina do Exercício e do Esporte.

\section{AGRADECIMENTOS}

O autor expressa o seu agradecimento à colaboração dos doutorandos Walace D. Monteiro e Marcos B. Almeida no manuseio do banco de dados do Flexiteste.
5. Araújo CGS. Flexiteste - Uma nova versão dos mapas de avaliação. Kinesis 1986;2:251-67.

6. Carvalho ACG, Paula KC, Azevedo TMC, Nóbrega ACL. Relação entre flexibilidade e força muscular em adultos jovens de ambos os sexos. Rev Bras Med Esporte 1998;4:2-8.

7. Farinatti PTV, Araújo CGS, Vanfraechem JHP. Influence of passive flexibility on the ease for swimming learning in pre-pubescent and pubescent children. Science et Motricité 1997;31:16-20. 
8. Araújo CGS. Avaliação e treinamento da flexibilidade. In: Ghorayeb N, Barros Neto TL, editores. O Exercício. São Paulo: Atheneu, 1999:25-34.

9. Araújo CGS. Flexitest - An office method for evaluation of flexibility. Sports \& Medicine Today 2001;1:34-7.

10. Araújo CGS. Correlação entre diferentes métodos lineares e adimensionais de avaliação da mobilidade articular. Rev Bras Ciên Mov 2000;8: 25-32.

11. Coelho CW, Araújo CGS. Relação entre aumento da flexibilidade e facilitações na execução de ações cotidianas em adultos participantes de programa de exercício supervisionado. Revista Brasileira de Cineantropometria \& Desempenho Humano 2000;2:31-41.

12. Silva LPS, Palma A, Araújo CGS. Validade da percepção subjetiva na avaliação da flexibilidade de adultos. Rev Bras Ciên Mov 2000;8:15-20.
13. Farinatti PTV, Nóbrega ACL, Araújo CGS. Perfil da flexibilidade em crianças de 5 a 15 anos de idade. Horizonte 1998;14:23-31.

14. Farinatti PTV, Soares PPS, Vanfraechem JHP. Influence de deux mois d'activités physiques sur la souplesse de femmes de 61 à 83 ans à partir d'un programme de promotion de la santé. Sport 1995;4:36-45.

15. Araújo CGS, Monteiro WD, Farinatti PTV. Body flexibility profile and clustering among elite athletes and age/gender-matched non-athletic population. Med Sci Sports Exerc 1999;31(Suppl 5):S115 (abstract).

16. Beighton $\mathrm{P}$, Horan F. Dominant inheritance in familial generalized articular hypermobility. J Bone Joint Surg [Br] 1970;52:145-59.

17. Rosenbloom AL, Silverstein JH, Lezote DC, Richardson K, McCallum M. Limited joint mobility in childhood diabetes mellitus indicates increased risk for microvascular disease. N Engl J Med 1981;305:191-4. 